\title{
Measuring Ferroelectric Order Parameters at Domain Walls and Vortices in Hexagonal Manganites with Atomic Resolution STEM
}

\author{
Megan E. Holtz ${ }^{1}$, Konstantin Shapovalov ${ }^{2}$, Julia A. Mundy ${ }^{1}$, Celesta S. Chang ${ }^{3}$, Dennis Meier ${ }^{4}$, Andres \\ Cano $^{2}$ and David A. Muller ${ }^{1}$ \\ 1. School of Applied and Engineering Physics, Cornell University, Ithaca, NY, USA \\ 2. CNRS, Université de Bordeaux, ICMCB, UPR 9048, Pessac, France \\ 3. Department of Physics, Cornell University, Ithaca, NY, USA \\ 4. Department of Materials Science and Engineering, Norwegian University of Science and Technology, \\ 7491 Trondheim, Norway
}

Hexagonal manganites exhibit a rich variety of topological defects, including vortices and neutral and charged domain walls. These topological defects are promising avenues for information carriers in nextgeneration memory devices. Here, we explore the atomic scale structure underlying ferroelectricity in hexagonal $\mathrm{ErMnO}_{3}$ (Fig 1a) with STEM. We quantify the local polarization by mapping the picometer scale Er buckling across ferroelectric domain walls and vortices. We measure the change in order parameter at charged and neutral walls and at vortices, and find a common length scale that is intrinsic to all of the topological defects within the Landau theory framework.

Key to mapping the order parameter is the ability to measure the picometer scale displacements that generate the polarization. The ferroelectric distortions in h-ErMnO 3 manifest as Er displacements in either an $\uparrow \uparrow \downarrow$ or $\downarrow \downarrow \uparrow$ pattern, with positive $(+P)$ or negative $(-P)$ polarization, which can be measured in HAADF-STEM along the [110] zone axis. Each polarization has three permutations, leading to six structural domains.[1] The order parameter is $(Q \cos \Phi, Q \sin \Phi)$, where $Q$ is the amplitude of the distortion and $\Phi_{n}=n \pi / 3$ is the phase, Fig 1b. STEM images were formed by many fast acquisitions in a $100 \mathrm{keV}$ NION Ultra-STEM, with a large beam current and stable stage, enabling location of the Er columns with $\sim 2$ pm precision.[2] We measure $Q$ and $\Phi$ over $10^{4} \mathrm{~nm}^{2}$, sampling the bulk, domain walls, and vortices. Fig. 1c shows six maxima in the occurrences of $Q$ and $\Phi$ displayed in order parameter space corresponding to the six domains, with a finite population of intermediate states with $\Phi \neq \mathrm{n} \pi / 3$.

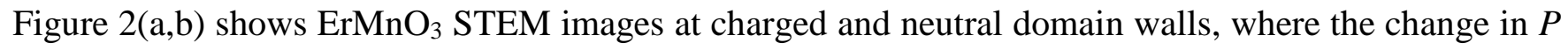
is transverse and parallel to the domain walls, respectively. The STEM images are overlaid with the color corresponding to the locally measured phase. In Fig. 2c, we aggregate $Q$ and $\Phi$ across many domain walls and fit using Landau theory (solid lines) to find the fundamental domain wall width, $\xi_{6}$. The gradual evolution across the domain wall is the origin of the intermediate states in Fig 1c. The charged domain wall is only slightly larger than the neutral walls, indicating that mechanical trimerization stiffness, rather than electrostatic effects, determine domain wall width. Figure 3 a shows a STEM image of a vortex in $\mathrm{Er}_{1-\mathrm{x}} \mathrm{Zr}_{\mathrm{x}} \mathrm{MnO}_{3}$, where six domains with different $\Phi$ merge. The amplitude $Q$ (Fig. 3b) drops to zero at the vortex central point and recovers the bulk value within a distance of $\sim 22 \AA$ (Fig. 3c). Our picometer mapping by STEM enables measurement of the change in order parameter at topological defects, giving insight into the fundamental physics behind these novel states of matter. [3]

\section{References:}

[1] H Das, et al, Nat. Comm. 5 (2014) 2889.

[2] A Yankovich et al, Nat. Comm. 5 (2014) 4155.

[3] Work supported by the U.S. DOE BES, Award \#DE-SC0002334. EM Facility support from the NSF MRSEC program (DMR 1120296). 

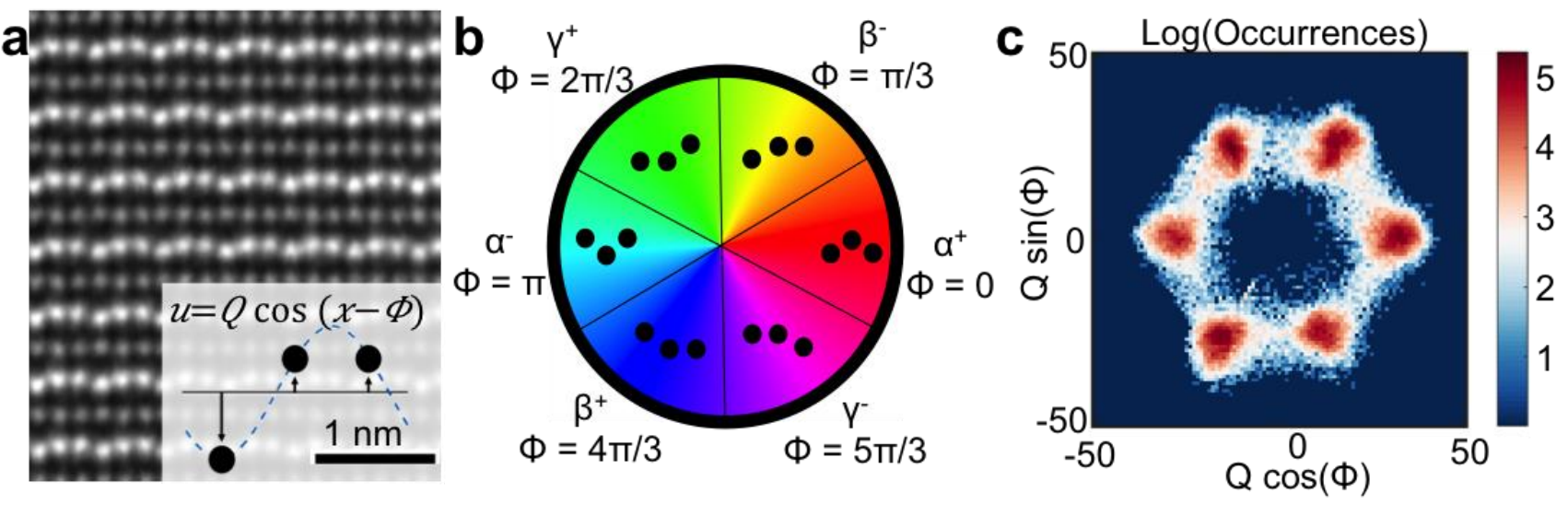

Figure 1. (a) STEM image showing ferroelectric rumpling. (b) Schematic of domains. (c) Histogram of occurrences of the order parameters, $\mathrm{Q}$ and $\Phi$, showing the six domains and intermediate states.
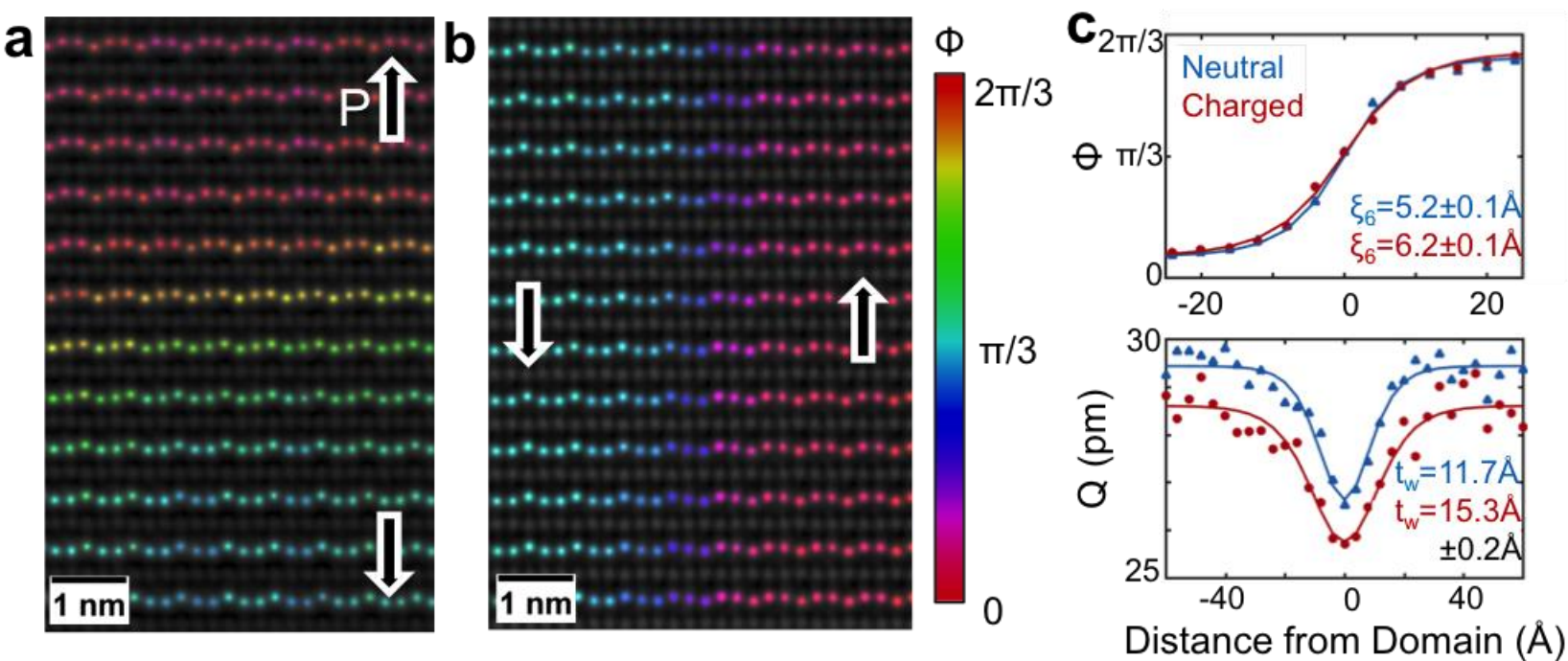

Figure 2. STEM images of (a) charged and (b) neutral domain walls, with color overlays corresponding to phase. (c) aggregated data of $\Phi$ and $Q$ across domain walls, showing slightly larger charged walls.

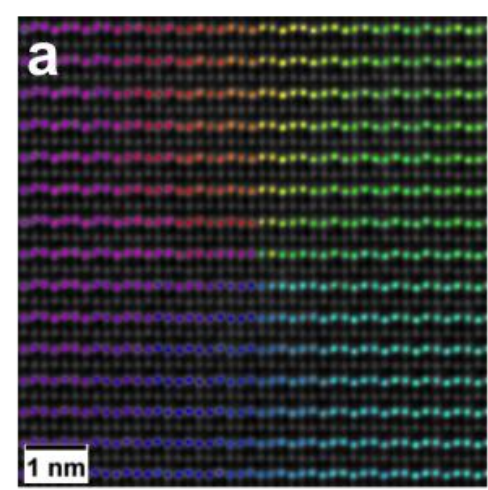

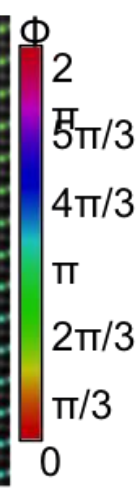

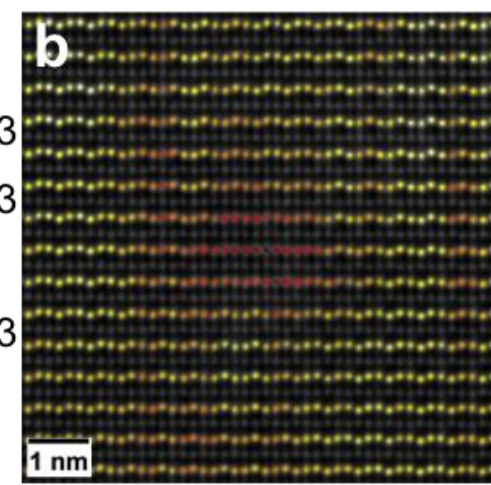

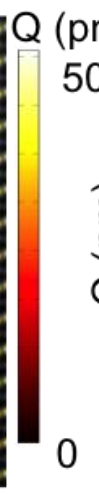

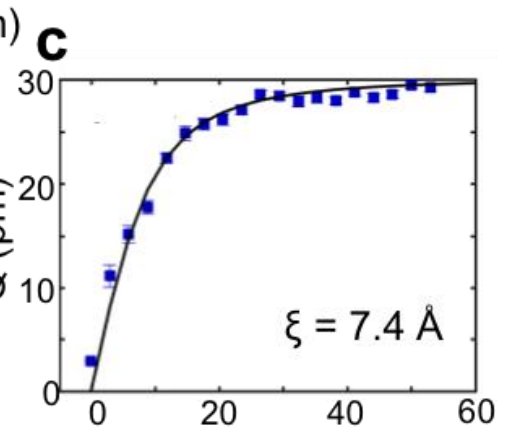

Radial distance from vortex

$(\AA)$

Figure 3. STEM images of a vortex with (a) phase color overlay and (b) amplitude overlay. (c) Q as a function of radial distance from vortex center, dropping to zero at vortex core. 